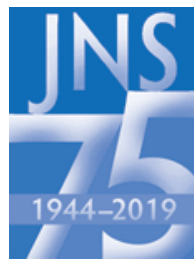

\title{
The intersect of neurosurgery with diffuse intrinsic pontine glioma
}

\author{
JNSPG 75th Anniversary Invited Review Article
}

Claudia M. Kuzan-Fischer, MD, ${ }^{1}$ and Mark M. Souweidane, MD'-3

Departments of ${ }^{1}$ Neurological Surgery and ${ }^{2}$ Pediatrics, Weill Cornell Medical College, NewYork-Presbyterian Hospital, New York; and ${ }^{3}$ Department of Neurological Surgery, Memorial Sloan Kettering Cancer Center, New York, New York

\begin{abstract}
An invited article highlighting diffuse intrinsic pontine glioma (DIPG) to celebrate the 75th Anniversary of the Journal of Neurosurgery, a journal known to define surgical nuance and enterprise, is paradoxical since DIPG has long been relegated to surgical abandonment. More recently, however, the neurosurgeon is emerging as a critical stakeholder given our role in tissue sampling, collaborative scientific research, and therapeutic drug delivery. The foundation for this revival lies in an expanding reliance on tissue accession for understanding tumor biology, available funding to fuel research, and strides with interventional drug delivery.
\end{abstract}

https://thejns.org/doi/abs/10.3171/2019.5.PEDS18376

KEYWORDS brainstem glioma; diffuse pontine glioma; DIPG; biopsy; surgery; oncology

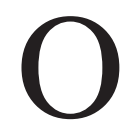
VER the years, multimodal treatment approaches have significantly contributed to improved survival for children with CNS tumors, and overall survival is at least 5 years for more than $70 \%$ of these patients. ${ }^{66}$ However, select tumor subtypes within this heterogeneous group of tumors, such as diffuse intrinsic pontine gliomas (DIPGs), continue to have a dismal prognosis. This discordance in progress with therapeutic benefit continues to frustrate the pediatric neuro-oncology community. Brainstem tumors are prevalent across the entire age spectrum; however, they occur predominantly in the pediatric population, accounting for $13.3 \%$ of all primary CNS tumors in the age group of $0-14$ years. ${ }^{49}$ There is a peak in incidence within a relatively narrow age range between 5 and 10 years and without gender bias. ${ }^{71}$ About $90 \%$ of brainstem tumors are gliomas. ${ }^{53}$ In children, the large majority are DIPGs, accounting for about $80 \%$ of all brainstem gliomas, ${ }^{29}$ which corresponds to $200-300$ new patients with this diagnosis in the United States each year. ${ }^{23,74}$

Due to extensive infiltration of the brainstem, the current standard of care for pediatric DIPG patients is local irradiation with a total dose of 54-60 Gy as well as treatment of hydrocephalus and tissue sampling for diagnosis and molecular tumor profiling whenever feasible. The ma- jority of patients show some transient clinical improvement and an extended survival of approximately 3 months in response to irradiation and steroid treatment. ${ }^{27}$ No further survival benefit has been demonstrated with the administration of radio-sensitizing agents ${ }^{8}$ or increased radiation doses up to $78 \mathrm{~Gy} .{ }^{50}$ DIPG patients commonly present with clinical or radiographic tumor progression approximately 3-8 months after completion of radiotherapy and many patients receive adjuvant chemotherapy within a clinical trial at this time. However, no neoadjuvant chemotherapeutic agent ${ }^{19}$ or high-dose chemotherapy with stem cell rescue $^{10}$ has proven any survival advantages.

DIPG continues to be a major therapeutic challenge and no significant improvement of survival has been accomplished over several decades despite the incorporation of proven therapeutic strategies in other pediatric CNS tumors. ${ }^{74}$ With a median survival of less than 12 months from diagnosis, ${ }^{15,26,36}$ DIPG remains the leading cause of brain tumor death in the pediatric population. ${ }^{36}$

\section{Neurosurgical Folklore}

Over the past 75 years, the neurosurgical intersect with DIPG has been characterized by a fluctuating process governed by surgical intent and counterbalanced by feasibil-

ABBREVIATIONS BBB = blood-brain barrier; $\mathrm{CED}$ = convection-enhanced delivery; DIPG = diffuse intrinsic pontine glioma; MRgFUS = MR-guided focused ultrasound . SUBMITTED April 5, 2019. ACCEPTED May 2, 2019.

INCLUDE WHEN CITING DOI: 10.3171/2019.5.PEDS18376. 


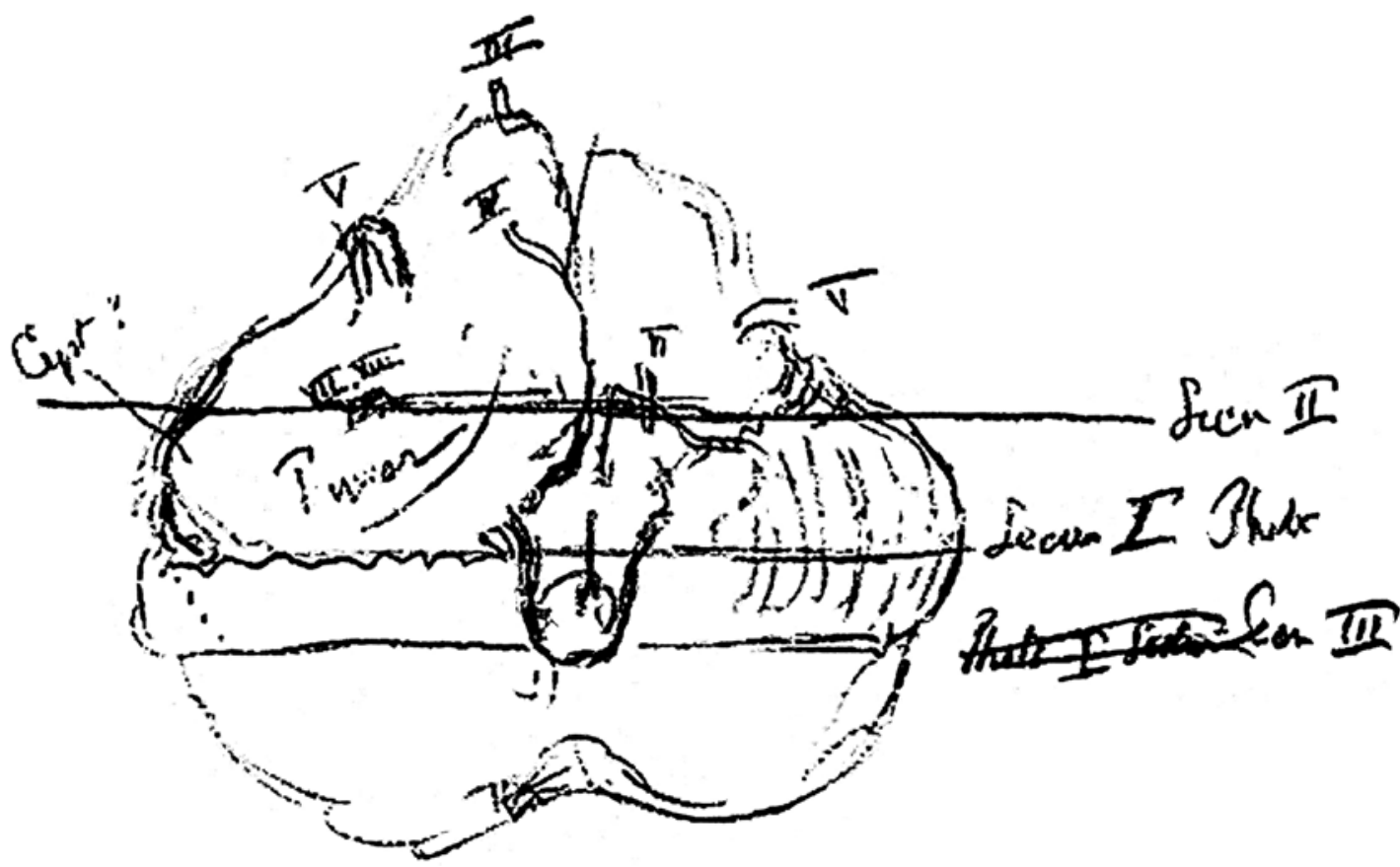

FIG. 1. Dr. Harvey Cushing's rendering of the postmortem brainstem section of a patient harboring a brainstem tumor. The diagram reinforces the neurosurgical pioneer's sentiment of inoperability. Used with permission. Courtesy of The Alan Mason Chesney Medical Archives of The Johns Hopkins Medical Institutions.

ity. Longer than the discipline of neurosurgery, the pontine segment of the brainstem has rightfully been recognized as a domain that is intolerant to surgery (Fig. 1). In fact, during the inception of our profession, cautionary notes and even forebodings guided the surgical landscape. In no uncertain terms, Harvey Cushing waved the flag of surgical abandonment for children with DIPG by stating, "I would like to see the day when somebody would be appointed surgeon somewhere who had no hands, for the operative part is the least part of the work" (H. Cushing 1911) ${ }^{17}$ This futility of surgical involvement was promulgated well into the 20th century. Dr. Donald Matson in 1969 reflected on brainstem gliomas as "malignant tumors since their location in itself renders them inoperable." ${ }^{44}$ Surgical inaccessibility is far from the only reason dissuading neurosurgical interest. Universal lethality, parental grief, practitioner's sense of inadequacy, a lack of DIPG-directed funding, and career futility all contribute to a dreadful environment that is easier avoided. DIPG is understandably anathema for generations of neurosurgeons.

\section{Controversies in Stereotactic Biopsy}

Historically, stereotactic biopsy of brainstem tumors is an old procedure and was first described by Gleason et al. in 1978. ${ }^{25}$ Limitations in diagnostic accuracy with CT in concert with the emergence of stereotactic-guided surgery led toward a rebirth of the surgeon's role with brainstem glioma. Both supratentorial and infratentorial stereotactic needle biopsy for lesions in the metencephalon carried an acceptable risk profile that legitimized the advocacy of tissue sampling. ${ }^{43,48}$

In the early 1990s, once again, the tide would shift with advanced imaging. MRI paved a path away from a surgical mindset. The anatomical resolution of MRI provided a very accurate estimation of clinical behavior. In fact, categories of brainstem tumors were largely born out of MRI-defined anatomical features. ${ }^{5,21}$

Generally, brainstem tumors are divided into focal and diffuse growing tumors. Further subdivision is based on location of origin (mesencephalon, pons [metencephalon], or medulla [myelencephalon]), extent and direction of tumor growth, exophytic or intrinsic growth pattern, and the presence or absence of contrast enhancement, hemorrhage, necrosis, cysts, and/or hydrocephalus. ${ }^{5,55}$ Based on MRI characteristics, Epstein and Farmer suggested 4 distinct subgroups of brainstem tumors: focal, dorsally exophytic, cervicomedullary, and diffusely infiltrating brainstem tumors. ${ }^{20}$

It became evident that MRI features could provide a highly reliable correlation with histological phenotype and the ability to reliably discriminate juvenile pilocytic astrocytoma from infiltrating astrocytomas (WHO grades II-IV). It would quickly be realized, however, that, regardless of the assigned grade according to the WHO, infiltrating (i.e., nonpilocytic) astrocytic tumors in the brainstem possessed a unifying clinical course of unremitting growth and ultimate death.

On MRI, DIPGs are characteristically hypo- to isointense on T1-weighted sequences and hyperintense on T2weighted imaging ${ }^{42}$ (Fig. 2A and C). On FLAIR sequences, DIPGs usually appear homogeneously hyperintense (Fig. 2D). ${ }^{74}$ The center of the tumor is typically located in the pons involving $>50 \%$ of the axial diameter. Other MRI features of typical DIPG are nondelineated tumor margins and engulfment of the basilar artery. ${ }^{71}$ Interest- 

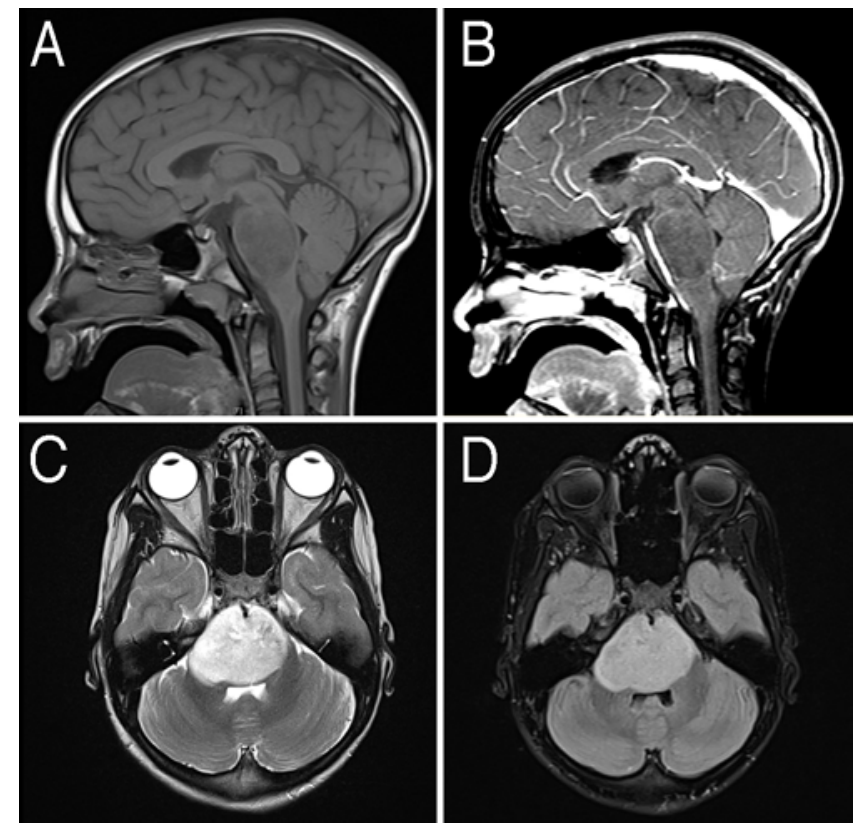

FIG. 2. Sagittal T1-weighted (A), sagittal T1-weighted postcontrast (B), axial T2-weighted (C), and axial FLAIR (D) MR images showing imaging features typical of DIPG.

ingly, there is usually no significant enhancement of these lesions on T1-weighted postcontrast sequences at the time of diagnosis ${ }^{28}$ (Fig. 2B). This suggests preservation of the blood-brain barrier (BBB), which is not in accordance with other high-grade gliomas. To a certain degree, it may reveal why chemotherapeutic drugs that have shown efficacy in the treatment of other high-grade gliomas have failed in the treatment of DIPG. ${ }^{42}$

The diagnosis of DIPG is based on a typical clinical presentation in the context of the above-described characteristic MRI findings. Classic findings on clinical examination of DIPG patients include the triad of cerebellar symptoms (ataxia, dysmetria, and/or dysarthria), long tract impairment (sensory loss, increased tone, decreased strength, clonus, Babinski sign, and/or hyperreflexia), and cranial nerve palsy (face asymmetry, diplopia due to impaired extraocular movement). ${ }^{35}$ Less than $10 \%$ of patients with DIPG present with hydrocephalus due to expansion of the pons or exophytic tumor growth leading to obstruction of the fourth ventricle. ${ }^{71}$ Other unspecific symptoms such as behavioral changes, school difficulties, and sleep apnea may occur at the time of diagnosis or later during the course of the disease. Typically, there is a short latency between onset of symptoms and time of diagnosis. ${ }^{74}$

This predictive capacity logically attenuated the desire or need for tissue sampling. ${ }^{2,51}$ For the next several decades, treatment of children with DIPG was predicated on a careful clinical history supplemented with MRI.

Stereotactic biopsy of DIPG patients has since been controversial and limited to patients with atypical presentation on MRI and more recently to patients enrolled in clinical trials at dedicated cancer centers. In 2011, Hankinson et al. described controversial results of a study asking pediatric neurosurgeons to decide whether the MRI characteristics of selected tumors were typical or atypical, and it was found that more than $75 \%$ of the participating neurosurgeons were in agreement of the MRI findings in only $43.8 \%$ of cases. ${ }^{28}$ Therefore, Hankinson et al. concluded that the diagnosis of a DIPG solely based on imaging features and a typical clinical presentation may not be sufficiently reliable as standard of care.

Compounding diagnostic uncertainty, the need for molecular phenotyping would be another defining element in the neurosurgeons' evolving role in DIPG management. As oncology transitioned into a biological era and genomic sequencing became more commonplace, desperate calls for obtaining biological DIPG material logically surfaced. In this era, medulloblastoma became the protoypical tumor in which molecular characterization would define and even supplant histological phenotype in prognostic and therapeutic planning. A parallel effort was not historically possible for malignant brainstem glioma since cytoreductive surgery was never integral to the clinical management of these children. Not only did this lack of tissue stagnate genomic evaluation, but it also hampered the development of experimental therapeutics. ${ }^{26,54}$ Clinical trials would therefore erroneously lump DIPG patients into clinical cohorts defined solely by a standard cytopathology interpretation. ${ }^{7}$ In the recent past, the urgency to improve the survival of patients with this universally fatal disease and the development of new molecular techniques have bolstered the role of stereotactic biopsy in DIPG patients in which scarce tissue would be accessible..$^{38,56}$

Meta-analyses of stereotactic biopsies in patients with brainstem lesions provided the field with insight and knowledge with regard to safety and diagnostic yield of this procedure. Most of these studies, however, include mixed population groups (children and adults) with a wide range of diagnoses. Overall, a postoperative morbidity of $0 \%-20 \%$ and a postoperative mortality of $0 \%-3 \%$ was reported within these studies. ${ }^{54}$ In 2015, Puget et al. published their Necker Enfants Malades Hospital series that describes the results of stereotactic biopsy in 130 children (median age 6.7 years, age range 16 months to 16.4 years) with typical imaging features of DIPG on MRI. ${ }^{54}$ The team performed a transcerebellar stereotactic biopsy in the prone position for all patients, and the stereotactic coordinates were defined preoperatively on CT or MRI with the goal of targeting a contrast-enhancing area of the lesion whenever feasible. In the beginning of the study, a single trajectory was chosen, and the specimen was collected using a Leksell stereotactic frame. Over time, the number of specimens was increased due to the potential intratumoral heterogeneity, as described by Buczkowicz et al. ${ }^{11}$ In doing so, the higher number of trajectories was not associated with increased risk of postoperative morbidity. Overall, transient neurological deficits were observed in $3.9 \%$ of patients ( 5 of 130 ), and the rate of persistent neurological deficit and mortality was $0 \%$ which demonstrated that stereotactic biopsy of DIPG is approximately as safe as supratentorial biopsy. ${ }^{54}$

Therefore, Puget et al. recommended offering stereotactic biopsy to all patients with DIPG in order to obtain tissue for molecular biology and genetic analyses and to identify new biological markers. ${ }^{54}$ Patient stratification 


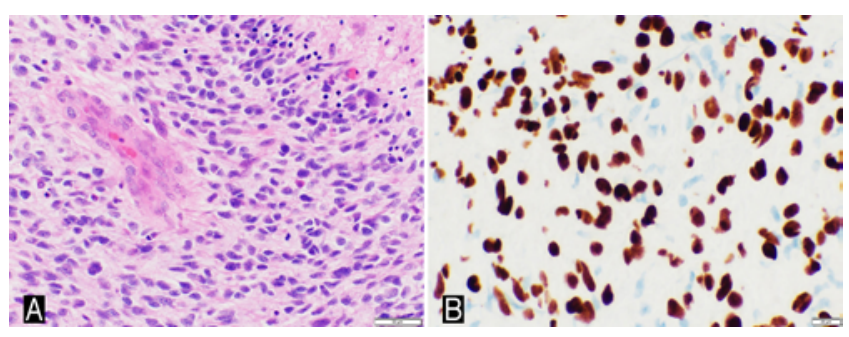

FIG. 3. A: An H \& E-stained section of pontine biopsy tissue, demonstrating a highly cellular population of atypical, spindled glial cells. Necrosis (upper right), microvascular proliferation (center left), and mitotic activity (lower center right) are evident. Original magnification $\times 400$; bar $=50 \mu \mathrm{m}$. B: Immunohistochemical staining using an antibody against the epitope containing the mutated H3 K27M residue, demonstrating strong positive nuclear labeling (brown staining), confirming the presence of H3F3A K27M mutation (also sometimes annotated as K28 M). The blue hematoxylin-stained nuclei without H3 K27M labeling represent endothelial cells and nonneoplastic glial cells and serve as an internal negative control. Original magnification $\times 600$; bar $=20 \mu \mathrm{m}$.

based on the biology and the molecular characteristics of the tumor will open the door for targeted therapy in clinical trials, with the goal of improvement of overall survival of patients with DIPG. While this approach was scientifically rational, it did incite colorful debate relating to the ethics of tissue sampling in the absence of recognized therapeutic value. ${ }^{67}$

\section{Biological Deluge}

With access to fresh tumor samples, the molecular underpinnings and disease modeling would soon follow. Although DIPGs are histologically similar to WHO grade II-IV fibrillary astrocytomas of other areas of the brain (Fig. 3A), ${ }^{11}$ genome- and epigenome-wide tumor profiling studies from tissues obtained by stereotactic pretreatment biopsies have changed our understanding of DIPG biology and have offered new perspectives in characterization by redefining this unique tumor entity. ${ }^{35}$

The first major breakthrough in understanding the mutational landscape of DIPG was in 2012, when different research groups reported that the majority of therapy-naive DIPGs exhibit mutations in histone H3, which indicated that DIPGs are epigenetically distinct from pediatric and adult supratentorial high-grade glioma (Fig. 3B). ${ }^{37,75}$ The recurrent $\mathrm{K} 27 \mathrm{M}$ mutations in the histone $\mathrm{H} 3$ gene $H 3 F 3 A$ and less commonly in the $H I S T 1 H 3 B$ gene are highly specific for midline astrocytomas arising in the brainstem. ${ }^{37}$ $\mathrm{K} 27 \mathrm{M}$ histone 3 mutations were identified in DIPG with WHO grade II, III, and IV histology and are predictive for outcome. On the contrary, the WHO grade of DIPG does not correlate with survival, as it was shown that DIPGs with a WHO grade II histology harboring the K27M mutation in the histone $\mathrm{H} 3$ carry a dismal prognosis, comparable to the overall survival of a patient with a WHO grade IV GBM tumor, while the DIPG wild-type for the K27M mutation with a WHO grade III or IV histology shows a patient overall survival equivalent to that of a wild-type WHO low-grade astrocytoma. ${ }^{11}$ The updated 2016 WHO classification of CNS tumors moved to an integrated diag-
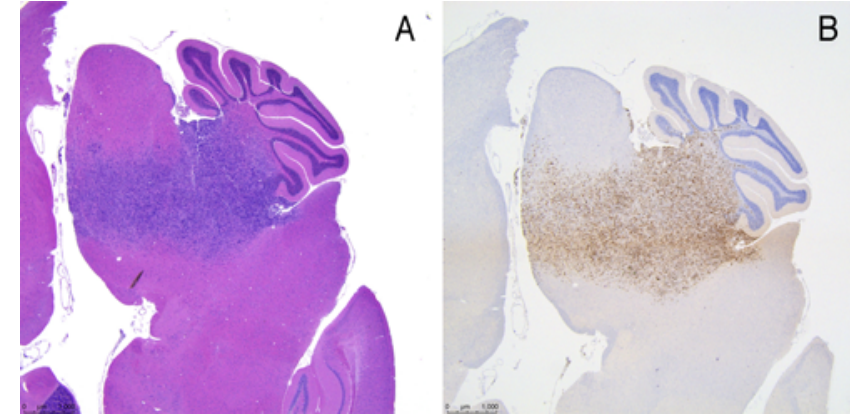

FIG. 4. Fixed formalin sections of DIPG xenograft showing $H$ \& $E(A)$ and Ki-67 (B) immunohistochemical staining, recapitulating the highly invasive capacity of these tumor models.

nosis, including histology, genetics, and epigenetics, which led to the new tumor category "diffuse midline glioma, H3 K27M-mutant." ${ }^{\circ}$

There has been an explosion in peer-reviewed publications on DIPG biology of late. Currently, sequencing is highly accessible, and doctors are having conversations with DIPG parents about tumor donation. Human-derived DIPG cell lines have been established, and expanding catalogs now exist for legitimate in vitro and in vivo preclinical research. Over the past decade, cell lines and murine models have been developed, more and more neuroscientists are studying the disease, and a dramatic change in our understanding of DIPG tumors has emerged (Figs. 4 and 5). Notably, the collaborative effort of the international research community is exemplified by a network of laboratories that are freely sharing these resources, thus expanding capacity and providing a method for validation.

One direct result of the DIPG genotyping has been the ability to generate unique, genetically engineered rodent models of DIPG. In 2017, Dr. Becher's group showed in a genetic DIPG mouse model that K27M mutation in the histone $\mathrm{H} 3$ variant $\mathrm{H} 3.3$ represses the tumor suppressor p16 and seems to play an important role in gliomagenesis, implicating that the G1-S cell-cycle transition may be a promising target for future therapeutic trials. ${ }^{16}$ The value of these biological substrates is also illustrated with the noteworthy findings of Drs. Monje and Mackall's groundbreaking work using CAR T-cell therapy in patient-derived H3 K27M-mutant glioma cell cultures as well as murine orthotopic xenografts. The authors were able to demonstrate potent antitumor efficacy of CAR T cells in diffuse midline gliomas with $\mathrm{K} 27 \mathrm{M}$ mutation in histone $\mathrm{H} 3$, and they identified GD2 as a new therapeutic target. ${ }^{47}$

These promising results show that the neuro-oncology research community is at the precipice of discovery with new therapeutic approaches that likely will translate into tangible hope.

\section{Translational Obstacles}

It has long been known that the BBB is a major challenge with respect to the delivery of chemotherapeutic agents for the treatment of brain tumors and consists of tight junctions along cerebral capillaries, protecting the CNS from toxins and bacteria. Most DIPGs present with 


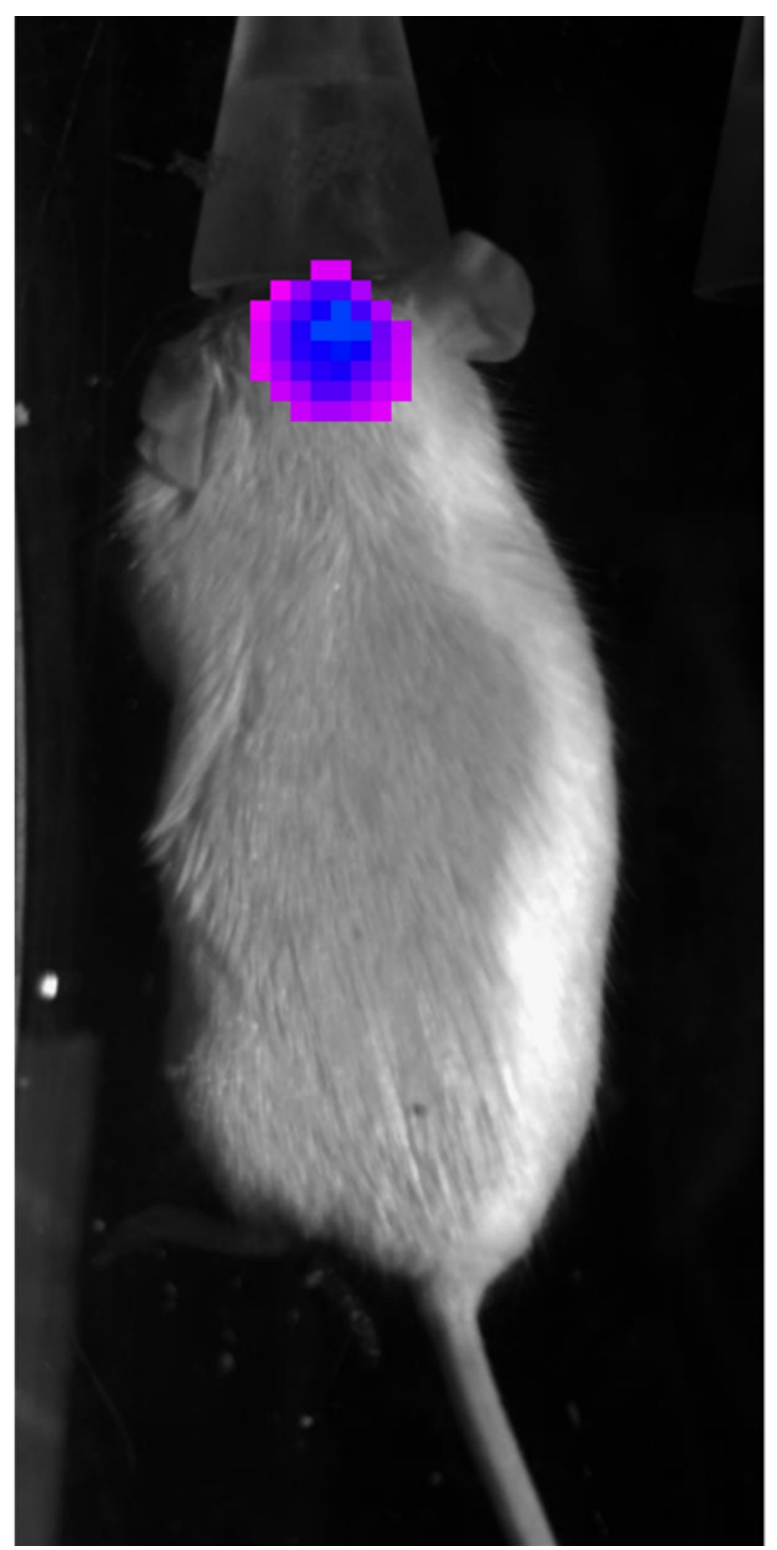

FIG. 5. Patient-derived orthotopic xenograft model of DIPG in a rodent. Human-derived cells include a reporter for bioluminescence. Bioluminescence imaging is used to monitor tumor burden.

insignificant contrast enhancement on MRI, implying that the BBB is relatively intact. The decades-long experience with negative results using adjuvant chemotherapy has buoyed concerns regarding pharmacokinetic drug availability within the DIPG tissue matrix. Convection enhanced delivery (CED), intraarterial drug delivery, and MR-guided focused ultrasound (MRgFUS) are contemporary neurosurgical interfaces that are in various phases of translational research.

\section{Enhanced Drug Delivery}

Enhanced drug delivery guides the motivation of several evolving neurosurgical procedures aimed at tumor treatment that are being explored in this disease.
Convection-enhanced delivery was first described in the $1990 \mathrm{~s}^{9}$ and has rapidly been introduced into the clinical arena following extensive preclinical testing. Using this modality, chemotherapeutic agents are delivered into the interstitial space of the tumor via microcatheter under continuous low hydraulic pressure. The technique affords many benefits compared with systemic delivery, most notably the ability to achieve high regional drug concentrations in the tissue of interest (on target) and near elimination of the drug from the systemic circulation (off target). So promising were preclinical efforts that the platform was quickly transitioned into clinical evaluation, most notably in the setting of multirecurrent supratentorial malignant glioma in adults. ${ }^{34}$ Early clinical experience would establish the safety or the procedural aspects and help refine device interfaces. Paralleling the clinical use of this promising drug delivery platform, however, was a lack of any established therapeutic benefit in overall survival even though objective responses were evident. These negative results highlight the importance of adequate dosimetry and drug delivery monitoring. Logically, therapeutic efficacy can only be achieved if drug distribution can be validated throughout a defined target volume. As an example, unsatisfactory efficacy resulting most likely from insufficient distribution of the infused agent was demonstrated in the phase 3 PRECISE study and in the TGFb-PE38 trial for glioblastoma. 57,58

Early on, we hypothesized that DIPG would provide a more appealing substrate for CED-based therapies (Fig. 6). Compared to most invasive gliomas, DIPG is relatively constrained based on volumetric features, thus predicting a greater likelihood of tumor coverage. Efficiency of largescale drug distribution with CED is negatively affected by features, including necrotic changes, cystic structures, and tissue inhomogeneities from past surgery, all uncommon features in DIPG patients. At diagnosis, DIPG patients are afflicted by disseminated disease at a much-reduced frequency compared with any other pediatric malignancies. Lastly, the desperate need for alternative therapeutic strategies for children with DIPG in some respects eases the regulatory process.

In 2002, our group demonstrated the feasibility and safety of CED within the brainstem in a rodent model. ${ }^{59}$ The safety and toxicity of potential therapeutic agents as well as the distribution pattern of the infusate have subsequently been studied in further animal studies ${ }^{41,62,63,65,69,70}$, 76,77 that have confirmed that CED does not cause relevant injury to the brainstem.

Early anecdotal experience in using CED as a means for drug delivery in children with DIPG was varied in surgical approach, device interface, therapeutic molecule, infusion parameters, and clinical condition. As one might expect, clinical tolerance was also varied and not unanimously supportive of further clinical application. Based on that limited experience, the feasibility of CED as a legitimate drug delivery platform for DIPG remained uncertain..$^{14}$ Clinical trials would soon follow that aimed to more concretely assess safety and define infusion parameters by using a systematic dose-escalation format. ${ }^{4,6,30}$ Our group was able to report comprehensively that CED in the brainstem of children with DIPG is a rational and reasonably 

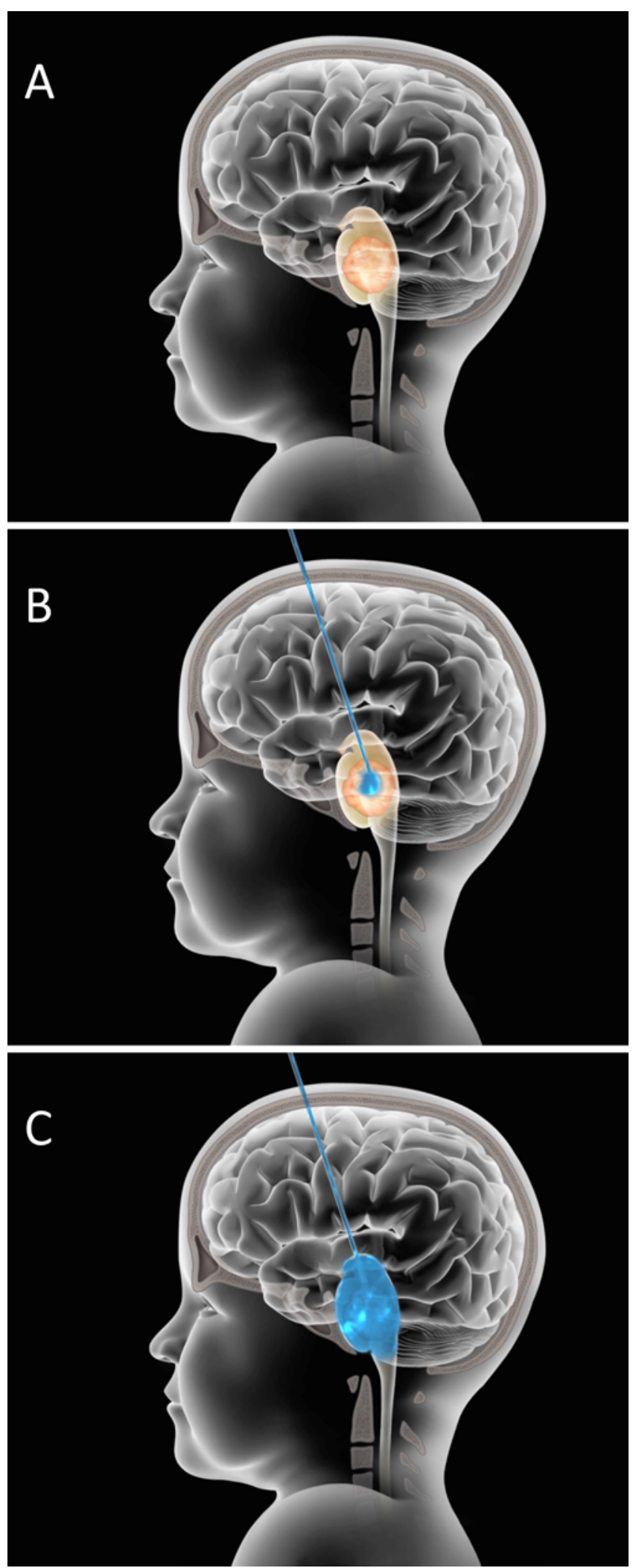

FIG. 6. The concept of using CED in a transfrontal MR-guided approach in DIPG is illustrated in this series of images obtained before treatment (A), during treatment (B), and at completion of treatment (C).
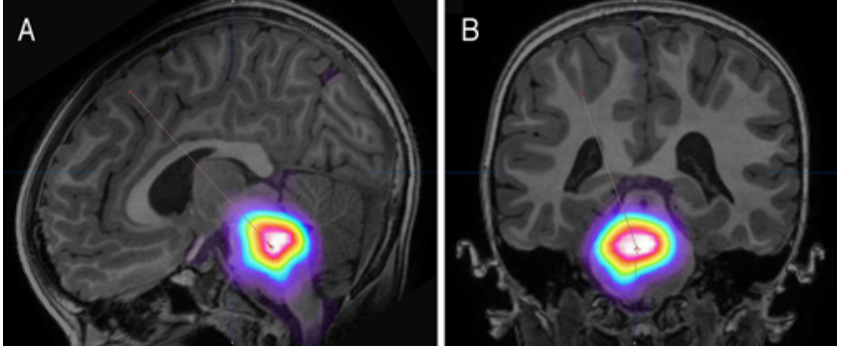

FIG. 7. Merged PET MR images obtained after infusion of a radioimmunotherapeutic (theranostic) molecule that provides quantitative measures of delivered dose. Sagittal (A) and coronal (B) sequences have color maps that correlate to the intensity of the PET signal.

safe treatment option. Twenty-eight patients were enrolled into a phase 1 clinical trial (registration no. NCT01502917, clinicaltrials.gov) and treated with a radiolabeled antibody and showed no dose-limiting toxicities. ${ }^{68}$ Besides the safety aspect, the study solidly validated the conceptual backbone of CED by demonstrating an over 1000-fold higher drug concentration in the intended pontine segment of the brainstem relative to the whole body (Fig. 7). ${ }^{68}$ Notably, single injections of approximately $4 \mathrm{ml}$ resulted in distribution volumes approximating estimations of tumor volume (Fig. 8). Current efforts are underway to explore this therapeutic drug delivery platform using a host of other therapeutic compounds, demonstrate multicenter feasibility, and define pharmacokinetic monitoring methods. Neurosurgeons are taking the lead to enhance device interfaces, validate drug distribution modeling, and assess robotic interfaces.

\section{MR-Guided Focused Ultrasound}

MR-guided focused ultrasound can be used for selective disruption of the $\mathrm{BBB} .{ }^{33}$ In order to reliably open the $\mathrm{BBB}$ without causing damage to the healthy brain tissue, albumin-coated microbubbles are injected. After intravenous injection, the microbubbles restrict the effects of the ultrasound to the vessel wall, ${ }^{32}$ which leads to selective disruption of the BBB even at lower acoustic power levels. ${ }^{45}$ As the duration of the BBB opening after microbubble-induced focused ultrasound lasts only about 5 hours ${ }^{72}$ repeated sonication may be required for repetitive or prolonged administration of an agent, which is a limitation of this modality. Based on the lack of ischemic or apoptotic changes in animal studies after focused ultrasound-induced BBB disruption, the modality seems to be relatively safe and not causing permanent damage to the healthy brain tissue. ${ }^{45}$ The safety of MRgFUS in opening the BBB in tumor patients is currently being evaluated in a clinical trial (registration no. NCT02343991, clinicaltrials. gov). Preclinical work in the brainstem has demonstrated that this noninvasive method can safely achieve greater concentrations of antineoplastic agents to the rodent brainstem compared with systemic delivery. ${ }^{3}$

\section{Intraarterial Drug Delivery}

Superselective intraarterial cerebral infusion is one mechanism through which we may increase the concentra- 


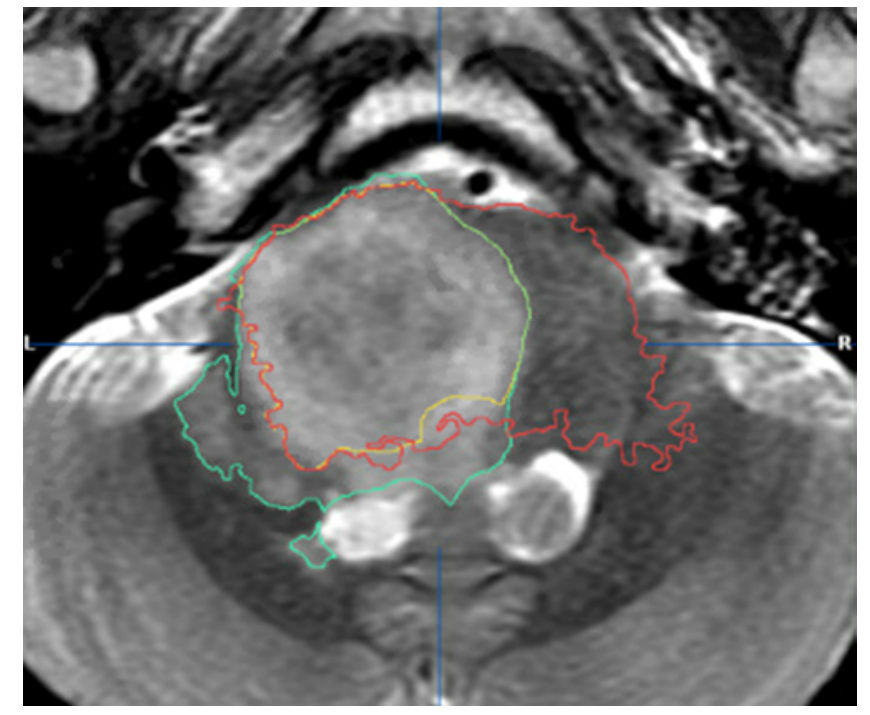

FIG. 8. Example transaxial MR image obtained in a patient treated with CED for a previously irradiated DIPG, demonstrating good creditable tumor coverage with therapy. The tumor volume is estimated based on T2 signal change prior to treatment (red outline), the distribution of the therapeutic compound is estimated based on a change in T2 signal compared with the preinfusion MR image (green outline), and the intersection of those volumes is calculated using a semiautomated algorithm (yellow outline).

tion of drugs within tumors through a combination of precisely opening the $\mathrm{BBB}$ and then delivering the drug to its target tissue during a permissive therapeutic window. This form of local drug delivery relies heavily on a defined arterial tributary for the anatomical site of interest. Clinical validation for this approach has been solidly demonstrated in infant rentinoblastoma. ${ }^{1,22,24,64}$ This backbone has resurfaced in treating supratentorial malignant glioma with the advent of superselective catheterization techniques. ${ }^{12,18}$ In the case of DIPG, the basilar artery is clearly the dominant arterial supply and superselective cannulation is relatively straightforward for a trained interventional neuroradiologist. Early-phase clinical trials (registration nos. NCT01688401 and NCT01884740, clinicaltrials.gov) are ongoing using this platform for children with DIPG, and results are expected to be forthcoming.

\section{No Simple Answer}

A legitimate concern related to loco-regional treatment (CED, MRgFUS, and intraarterial drug delivery) for DIPG is the known potential for CSF tumor dissemination or distant invasive growth patterns (Fig. 9). These features, commonly, but not always, are late features of the disease and introduce a degree of complexity when the platform is local ${ }^{61,73}$ While legitimate, these concerns are not absolute and should not interfere with further exploration, given the robust improvement in drug delivery capability. First, one must consider that a focal therapeutic approach, external-beam radiotherapy, has been the mainstay of therapy for several decades. This oncological acceptance alone supports the notion. Second, if regional control can be ultimately documented in DIPG, then vigorous efforts
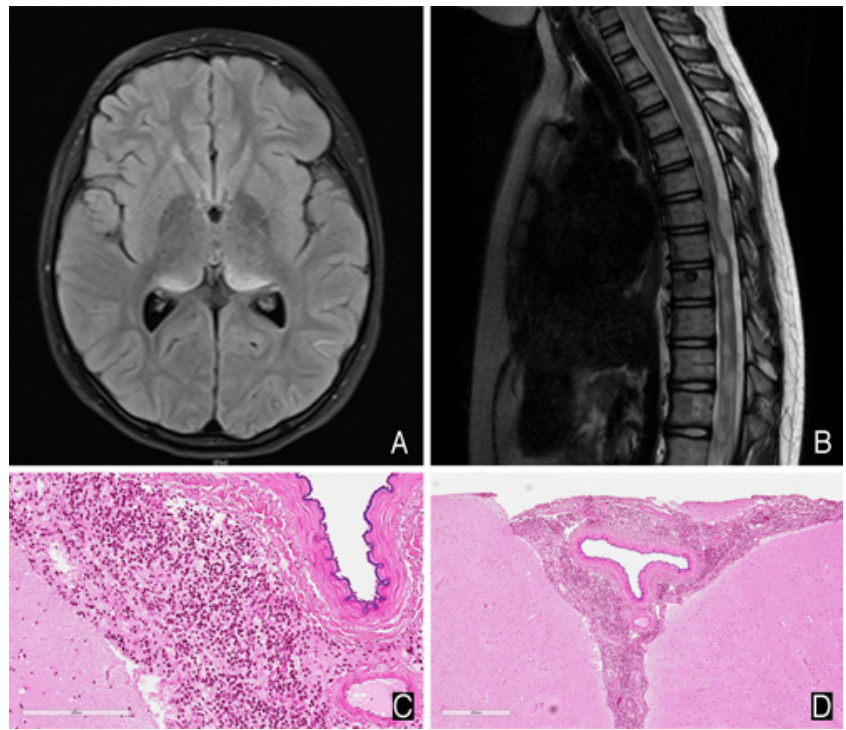

FIG. 9. A: Axial FLAIR sequence obtained in a DIPG patient with tumor dissemination as indicated by the bilateral FLAIR signal in the posterior thalami. B: Sagittal MR image obtained in a DIPG patient with numerous expansile nonenhancing T2 hyperintense intramedullary foci seen throughout the thoracic spinal cord, indicating tumor dissemination.

C and D: H \& E-stained sections obtained during a postmortem examination of a child who died of disseminated DIPG. Original magnification $\times 5$; bar $=500 \mu \mathrm{m}(C)$. Original magnification $\times 20$; bar $=200 \mu \mathrm{m}(\mathrm{D})$.

would need to be integrated into these regional strategies. As a parallel, nearly all therapeutic strategies for primary malignancies of the brain in children begin with aggressive local control by way of cytoreductive surgery. Local disease control using unconventional platforms such as CED thus might be viewed as a form of chemosurgery that imparts that same oncological benefit to gross-total tumor removal. It is likely that if regional control can be documented then supplementation with a traditional (i.e., craniospinal irradiation or systemic chemotherapy) or innovative (i.e., immune modulation or intrathecal targeted delivery) global approach might be needed. .1,39,52,60 $^{2}$

\section{Response Monitoring}

Historical controls focusing on survival rates, specifically overall survival, have served as the measure of therapeutic benefit in ongoing clinical trials and early-phase pilot studies in children with DIPG. This comparison is just, given the uniformly abysmal prognosis of the disease and minimal variance in outcome from one clinical trial to another. In general, the overall survival for patients with DIPG is less than 12 months from time of diagnosis. ${ }^{15,26,36}$ While this measure serves as a legitimate comparison, the validity of this needs to be taken in context. First, with several evolving therapies, particularly those that implement localized therapy (e.g., CED, MRgFUS), one can imagine that local control might be a more realistic measure of therapeutic benefit.

In addition to survival measures, early response monitoring is needed to better assess any therapeutic benefit within one individual. Biomarkers of response would be 
70

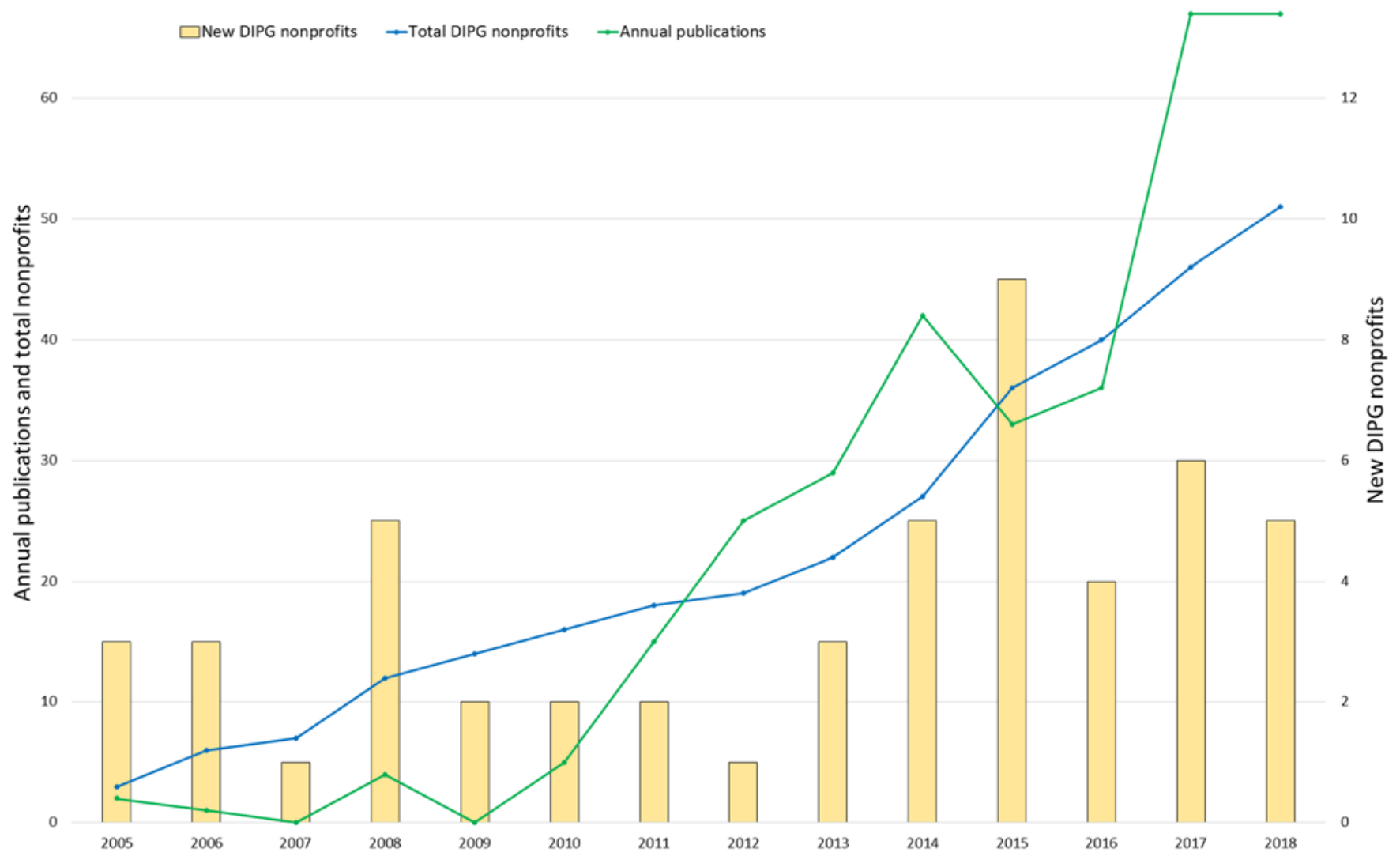

FIG. 10. The number of DIPG-directed funding through nonprofits and the number of peer-reviewed publications (PubMed search term "DIPG") has continuously increased over the past 15 years. The positive correlation between funding and published research supports the hypothesis that this funding has directly fueled meaningful biomedical and clinical research.

invaluable in monitoring response to experimental therapeutics and offer insight into the need for modifying or aborting a specific therapeutic approach. Liquid biopsy is a rapidly evolving technology being explored in the longitudinal assessment of primary glial neoplasms of the brain. ${ }^{46}$ Early reports of DIPG-specific application of liquid biopsy are encouraging. ${ }^{31,52,60}$ One can envision that longitudinal CSF sampling might impart demand on the neurosurgeon to implant access devices for near-continuous feedback.

\section{Fueling the Fervor}

One highly successful chapter related to DIPG has been the groundswell of available funding for preclinical and clinical research. In an era of diminishing federal funding for pediatric brain tumor research, compassionate persons affected and touched by grief have auspiciously forced DIPG out of obscurity and into a marquee status. While the total funds may be dwarfed by research dollars available for more common cancers, never before has there been such a collective effort to incite research in DIPG biology.

In the early 2000s, grants/funding specific to DIPG was negligible, and grant applications were not specific to the disease (sometimes not even specific to pediatric brain tu- mors). Not-for-profit foundations began to award research dollars for DIPG-specific programs aimed at improving our understanding of this disease. Individualized efforts, while strong in their intent, had little capacity to fund meaningful research. The request for the international research community to collaborate forced aggregation of intellect and resources that would propel DIPG into one of the best molecularly characterized brain tumors. In 2011, the DIPG Collaborative was launched, and funding was made available to use an international registry that would feed into a vibrant bioinformatics platform. Dr. Maryam Fouladi, professor and medical director of neuro-oncology at the Cincinnati Children's Hospital Medical Center, was awarded USD \$219,000 in January 2012 for her project, establishment of the International DIPG Registry. This registry is a comprehensive database and includes clinical, radiological, pathologic, and molecular data of DIPG patients. It was launched with the intention to create an international collaboration between researchers and clinicians with the overall goal to improve the outcome for patients with this fatal disease. In 2018, it was reported that 106 academic medical centers from 14 countries have been working collaboratively within the International DIPG Registry, contributing significantly to DIPG research. ${ }^{13}$

Since 2005, 51 families who have lost children to the disease have been approved for 501c3 nonprofit status in 
the US to fundraise in support of DIPG-specific research. As of 2018, those 51 family-founded charities are raising an estimated \$11 million per year to fuel research efforts. Demonstrative of this grassroots potential is the aggregate support funneled through the Pediatric Brain Tumor Project at Weill Cornell Medical College that has fueled the preclinical and subsequent translational work of the senior author (M.M.S.). The emergence of family-founded nonprofits, large and small, has expanded the number of new investigators and the number of projects being funded for DIPG around the world. Annually the number of DIPG nonprofits continues to rise, with a direct result in investigative vigor (Fig. 10). DIPG has now assumed a far more urgent priority on the pediatric neuro-oncology agenda as a result of these grassroots efforts. The propulsion of DIPG into the forefront of preclinical and clinical efforts is to be credited to the nonprofits as much as to the individuals on the forefront of discovery.

\section{The Neurosurgical Horizon}

The future role of the neurosurgeon in the management of DIPG is uncertain. What is likely, however, is that interventional therapeutic platforms and tissue sampling, both solid and liquid, will establish our role in the management of these unfortunate children. Neurosurgery must coalesce these efforts, contribute to device interface research and development, and attempt to standardize guidelines that incorporate objectives, indications, and safe processes.

\section{Acknowledgments}

We thank Dr. David J. Pisapia, Dr. Cynthia Hawkins, Cindy Campbell, and Umberto Tosi for contributing images. We also thank Thom Grave for his illustrations used in this paper. Nearly all the funding that has supported the translational and clinical efforts under the leadership of Dr. Souweidane has been received through generous gifts and grant awards by nonprofit organizations. The following list represents a sample, which is likely not exclusive, of these types of organizations: Alex's Lemonade Stand, Believe in Brooke, Children's Brain Tumor Family Foundation, Christian Koehler Foundation, Cookies for Kids' Cancer, Fly a Kite Foundation, Head for the Cure Foundation, Ian's Friends Foundation, Joshua's Wish, Lily LaRue Foundation, Louis and Rachel Rudin Foundation, Lyla Nsouli Foundation, Lyonhearted Foundation, McKenna Claire Foundation, Pediatric Brain Tumor Foundation, Pershing Square Foundation, Solving Kids' Cancer, St. Baldrick's Foundation, The Brooke Healey Foundation, The ChadTough Foundation, The Cristian Rivera Foundation, The Cure Starts Now Foundation, The Dana Foundation, The Matthew Larson Foundation for Pediatric Brain Tumors, The New York Community Trust, The Olivia Boccuzzi Foundation, and the Ty Louis Campbell Foundation.

\section{References}

1. Abramson DH, Dunkel IJ, Brodie SE, Kim JW, Gobin YP: A phase I/II study of direct intraarterial (ophthalmic artery) chemotherapy with melphalan for intraocular retinoblastoma initial results. Ophthalmology 115:1398-1404, 1404.e1, 2008

2. Albright AL: Tumors of the pons. Neurosurg Clin N Am 4:529-536, 1993

3. Alli S, Figueiredo CA, Golbourn B, Sabha N, Wu MY, Bon$\operatorname{doc} \mathrm{A}$, et al: Brainstem blood brain barrier disruption using focused ultrasound: a demonstration of feasibility and en- hanced doxorubicin delivery. J Control Release 281:29-41, 2018

4. Anderson RC, Kennedy B, Yanes CL, Garvin J, Needle M, Canoll P, et al: Convection-enhanced delivery of topotecan into diffuse intrinsic brainstem tumors in children. J Neurosurg Pediatr 11:289-295, 2013

5. Barkovich AJ, Krischer J, Kun LE, Packer R, Zimmerman RA, Freeman CR, et al: Brain stem gliomas: a classification system based on magnetic resonance imaging. Pediatr Neurosurg 16:73-83, 1990-1991

6. Barua NU, Lowis SP, Woolley M, O’Sullivan S, Harrison R, Gill SS: Robot-guided convection-enhanced delivery of carboplatin for advanced brainstem glioma. Acta Neurochir (Wien) 155:1459-1465, 2013

7. Bax DA, Mackay A, Little SE, Carvalho D, Viana-Pereira M, Tamber N, et al: A distinct spectrum of copy number aberrations in pediatric high-grade gliomas. Clin Cancer Res 16:3368-3377, 2010

8. Bernier-Chastagner V, Grill J, Doz F, Bracard S, Gentet JC, Marie-Cardine A, et al: Topotecan as a radiosensitizer in the treatment of children with malignant diffuse brainstem gliomas: results of a French Society of Paediatric Oncology Phase II Study. Cancer 104:2792-2797, 2005

9. Bobo RH, Laske DW, Akbasak A, Morrison PF, Dedrick RL, Oldfield EH: Convection-enhanced delivery of macromolecules in the brain. Proc Natl Acad Sci U S A 91:2076-2080, 1994

10. Bouffet E, Raquin M, Doz F, Gentet JC, Rodary C, Demeocq F, et al: Radiotherapy followed by high dose busulfan and thiotepa: a prospective assessment of high dose chemotherapy in children with diffuse pontine gliomas. Cancer 88:685-692, 2000

11. Buczkowicz P, Bartels U, Bouffet E, Becher O, Hawkins C: Histopathological spectrum of paediatric diffuse intrinsic pontine glioma: diagnostic and therapeutic implications. Acta Neuropathol 128:573-581, 2014

12. Chakraborty S, Filippi CG, Wong T, Ray A, Fralin S, Tsiouris AJ, et al: Superselective intraarterial cerebral infusion of cetuximab after osmotic blood/brain barrier disruption for recurrent malignant glioma: phase I study. J Neurooncol 128:405-415, 2016

13. Chaney B: International DIPG Registry Update: August 2018. DIPGRegistry.org (https://dipgregistry.org/international-dipg-registry-update-august-2018/) [Accessed May 9, 2019]

14. Chittiboina P, Heiss JD, Warren KE, Lonser RR: Magnetic resonance imaging properties of convective delivery in diffuse intrinsic pontine gliomas. J Neurosurg Pediatr 13:276282, 2014

15. Cohen KJ, Heideman RL, Zhou T, Holmes EJ, Lavey RS, Bouffet E, et al: Temozolomide in the treatment of children with newly diagnosed diffuse intrinsic pontine gliomas: a report from the Children's Oncology Group. Neuro Oncol 13:410-416, 2011

16. Cordero FJ, Huang Z, Grenier C, He X, Hu G, McLendon RE, et al: Histone H3.3K27M represses p16 to accelerate gliomagenesis in a murine model of DIPG. Mol Cancer Res 15:1243-1254, 2017

17. Dmetrichuk JM, Pendleton C, Jallo GI, Quiñones-Hinojosa A: Father of neurosurgery: Harvey Cushing's early experience with a pediatric brainstem glioma at the Johns Hopkins Hospital. J Neurosurg Pediatr 8:337-341, 2011

18. Doolittle ND, Miner ME, Hall WA, Siegal T, Jerome E, Osztie E, et al: Safety and efficacy of a multicenter study using intraarterial chemotherapy in conjunction with osmotic opening of the blood-brain barrier for the treatment of patients with malignant brain tumors. Cancer 88:637-647, 2000

19. Doz F, Neuenschwander S, Bouffet E, Gentet JC, Schneider $\mathrm{P}$, Kalifa $\mathrm{C}$, et al: Carboplatin before and during radiation 
therapy for the treatment of malignant brain stem tumours: a study by the Société Française d'Oncologie Pédiatrique. Eur J Cancer 38:815-819, 2002

20. Epstein FJ, Farmer JP: Brain-stem glioma growth patterns. J Neurosurg 78:408-412, 1993

21. Fischbein NJ, Prados MD, Wara W, Russo C, Edwards MS, Barkovich AJ: Radiologic classification of brain stem tumors: correlation of magnetic resonance imaging appearance with clinical outcome. Pediatr Neurosurg 24:9-23, 1996

22. Francis JH, Roosipu N, Levin AM, Brodie SE, Dunkel IJ, Gobin YP, et al: Current treatment of bilateral retinoblastoma: the impact of intraarterial and intravitreous chemotherapy. Neoplasia 20:757-763, 2018

23. Frazier JL, Lee J, Thomale UW, Noggle JC, Cohen KJ, Jallo GI: Treatment of diffuse intrinsic brainstem gliomas: failed approaches and future strategies. J Neurosurg Pediatr 3:259-269, 2009

24. Funes S, Sampor C, Villasante F, Fandiño A, Manzitti J, Sgroi $\mathrm{M}$, et al: Feasibility and results of an intraarterial chemotherapy program for the conservative treatment of retinoblastoma in Argentina. Pediatr Blood Cancer 65:e27086, 2018

25. Gleason CA, Wise BL, Feinstein B: Stereotactic localization (with computerized tomographic scanning), biopsy, and radiofrequency treatment of deep brain lesions. Neurosurgery 2:217-222, 1978

26. Grasso CS, Tang Y, Truffaux N, Berlow NE, Liu L, Debily MA, et al: Functionally defined therapeutic targets in diffuse intrinsic pontine glioma. Nat Med 21:827, 2015

27. Haas-Kogan DA, Banerjee A, Poussaint TY, Kocak M, Prados MD, Geyer JR, et al: Phase II trial of tipifarnib and radiation in children with newly diagnosed diffuse intrinsic pontine gliomas. Neuro Oncol 13:298-306, 2011

28. Hankinson TC, Campagna EJ, Foreman NK, Handler MH: Interpretation of magnetic resonance images in diffuse intrinsic pontine glioma: a survey of pediatric neurosurgeons. $\mathbf{J}$ Neurosurg Pediatr 8:97-102, 2011

29. Hargrave D, Bartels U, Bouffet E: Diffuse brainstem glioma in children: critical review of clinical trials. Lancet Oncol 7:241-248, 2006

30. Heiss JD, Jamshidi A, Shah S, Martin S, Wolters PL, Argersinger DP, et al: Phase I trial of convection-enhanced delivery of IL13-Pseudomonas toxin in children with diffuse intrinsic pontine glioma. J Neurosurg Pediatr 23:333-342, 2018

31. Huang TY, Piunti A, Lulla RR, Qi J, Horbinski CM, Tomita $\mathrm{T}$, et al: Detection of histone H3 mutations in cerebrospinal fluid-derived tumor DNA from children with diffuse midline glioma. Acta Neuropathol Commun 5:28, 2017

32. Hynynen K, McDannold N, Sheikov NA, Jolesz FA, Vykhodtseva N: Local and reversible blood-brain barrier disruption by noninvasive focused ultrasound at frequencies suitable for trans-skull sonications. Neuroimage 24:12-20, 2005

33. Hynynen K, McDannold N, Vykhodtseva N, Jolesz FA: Noninvasive MR imaging-guided focal opening of the bloodbrain barrier in rabbits. Radiology 220:640-646, 2001

34. Jahangiri A, Chin AT, Flanigan PM, Chen R, Bankiewicz K, Aghi MK: Convection-enhanced delivery in glioblastoma: a review of preclinical and clinical studies. J Neurosurg 126:191-200, 2017

35. Johung TB, Monje M: Diffuse intrinsic pontine glioma: new pathophysiological insights and emerging therapeutic targets. Curr Neuropharmacol 15:88-97, 2017

36. Kebudi R, Cakir FB: Management of diffuse pontine gliomas in children: recent developments. Paediatr Drugs 15:351362,2013

37. Khuong-Quang DA, Buczkowicz P, Rakopoulos P, Liu XY, Fontebasso AM, Bouffet E, et al: K27M mutation in histone H3.3 defines clinically and biologically distinct subgroups of pediatric diffuse intrinsic pontine gliomas. Acta Neuropathol 124:439-447, 2012
38. Kieran MW: Time to rethink the unthinkable: upfront biopsy of children with newly diagnosed diffuse intrinsic pontine glioma (DIPG). Pediatr Blood Cancer 62:3-4, 2015

39. Kossatz S, Carney B, Schweitzer M, Carlucci G, Miloushev VZ, Maachani UB, et al: Biomarker-based PET imaging of diffuse intrinsic pontine glioma in mouse models. Cancer Res 77:2112-2123, 2017

40. Louis DN, Perry A, Reifenberger G, von Deimling A, Figarella-Branger D, Cavenee WK, et al: The 2016 World Health Organization Classification of Tumors of the Central Nervous System: a summary. Acta Neuropathol 131:803-820, 2016

41. Luther N, Zhou Z, Zanzonico P, Cheung NK, Humm J, Edgar MA, et al: The potential of theragnostic ${ }^{124} \mathrm{I}-8 \mathrm{H} 9$ convectionenhanced delivery in diffuse intrinsic pontine glioma. Neuro Oncol 16:800-806, 2014

42. Mathew RK, Rutka JT: Diffuse intrinsic pontine glioma: clinical features, molecular genetics, and novel targeted therapeutics. J Korean Neurosurg Soc 61:343-351, 2018

43. Mathisen JR, Giunta F, Marini G, Backlund EO: Transcerebellar biopsy in the posterior fossa: 12 years experience. Surg Neurol 28:100-104, 1987

44. Matson DD, Ingraham FD: Neurosurgery of Infancy and Childhood, ed 2. Springfield, IL: Thomas, 1969

45. McDannold N, Vykhodtseva N, Raymond S, Jolesz FA, Hynynen K: MRI-guided targeted blood-brain barrier disruption with focused ultrasound: histological findings in rabbits. Ultrasound Med Biol 31:1527-1537, 2005

46. Miller AM, Shah RH, Pentsova EI, Pourmaleki M, Briggs $\mathrm{S}$, Distefano N, et al: Tracking tumour evolution in glioma through liquid biopsies of cerebrospinal fluid. Nature 565:654-658, 2019

47. Mount CW, Majzner RG, Sundaresh S, Arnold EP, Kadapakkam M, Haile S, et al: Potent antitumor efficacy of anti-GD2 CAR T cells in H3-K27M+ diffuse midline gliomas. Nat Med 24:572-579, 2018

48. Ostertag CB, Mennel HD, Kiessling M: Stereotactic biopsy of brain tumors. Surg Neurol 14:275-283, 1980

49. Ostrom QT, Gittleman H, Truitt G, Boscia A, Kruchko C, Barnholtz-Sloan JS: CBTRUS statistical report: primary brain and other central nervous system tumors diagnosed in the United States in 2011-2015. Neuro Oncol 20 (suppl_4):iv1-iv86, 2018

50. Packer RJ, Boyett JM, Zimmerman RA, Albright AL, Kaplan AM, Rorke LB, et al: Outcome of children with brain stem gliomas after treatment with $7800 \mathrm{cGy}$ of hyperfractionated radiotherapy. A Childrens Cancer Group Phase I/II Trial. Cancer 74:1827-1834, 1994

51. Packer RJ, Nicholson HS, Vezina LG, Johnson DL: Brainstem gliomas. Neurosurg Clin N Am 3:863-879, 1992

52. Pan C, Diplas BH, Chen X, Wu Y, Xiao X, Jiang L, et al: Molecular profiling of tumors of the brainstem by sequencing of CSF-derived circulating tumor DNA. Acta Neuropathol 137:297-306, 2019

53. Pierre-Kahn A, Hirsch JF, Vinchon M, Payan C, Sainte-Rose C, Renier D, et al: Surgical management of brain-stem tumors in children: results and statistical analysis of 75 cases. J Neurosurg 79:845-852, 1993

54. Puget S, Beccaria K, Blauwblomme T, Roujeau T, James S, Grill J, et al: Biopsy in a series of 130 pediatric diffuse intrinsic pontine gliomas. Childs Nerv Syst 31:1773-1780, 2015

55. Recinos PF, Sciubba DM, Jallo GI: Brainstem tumors: where are we today? Pediatr Neurosurg 43:192-201, 2007

56. Robison NJ, Kieran MW: Diffuse intrinsic pontine glioma: a reassessment. J Neurooncol 119:7-15, 2014

57. Sampson JH, Akabani G, Archer GE, Berger MS, Coleman $\mathrm{RE}$, Friedman $\mathrm{AH}$, et al: Intracerebral infusion of an EGFRtargeted toxin in recurrent malignant brain tumors. Neuro Oncol 10:320-329, 2008 
58. Sampson JH, Archer G, Pedain C, Wembacher-Schröder E, Westphal M, Kunwar S, et al: Poor drug distribution as a possible explanation for the results of the PRECISE trial. J Neurosurg 113:301-309, 2010

59. Sandberg DI, Edgar MA, Souweidane MM: Convectionenhanced delivery into the rat brainstem. J Neurosurg 96:885-891, 2002

60. Saratsis AM, Yadavilli S, Magge S, Rood BR, Perez J, Hill DA, et al: Insights into pediatric diffuse intrinsic pontine glioma through proteomic analysis of cerebrospinal fluid. Neuro Oncol 14:547-560, 2012

61. Sethi R, Allen J, Donahue B, Karajannis M, Gardner S, Wisoff J, et al: Prospective neuraxis MRI surveillance reveals a high risk of leptomeningeal dissemination in diffuse intrinsic pontine glioma. J Neurooncol 102:121-127, 2011

62. Sewing AC, Caretti V, Lagerweij T, Schellen P, Jansen MH, van Vuurden DG, et al: Convection enhanced delivery of carmustine to the murine brainstem: a feasibility study. J Neurosci Methods 238:88-94, 2014

63. Sewing ACP, Lagerweij T, van Vuurden DG, Meel MH, Veringa SJE, Carcaboso AM, et al: Preclinical evaluation of convection-enhanced delivery of liposomal doxorubicin to treat pediatric diffuse intrinsic pontine glioma and thalamic high-grade glioma. J Neurosurg Pediatr 19:518-530, 2017

64. Shields CL, Kaliki S, Al-Dahmash S, Rojanaporn D, Leahey A, Griffin G, et al: Management of advanced retinoblastoma with intravenous chemotherapy then intra-arterial chemotherapy as alternative to enucleation. Retina 33:2103-2109, 2013

65. Singleton WGB, Bienemann AS, Woolley M, Johnson D, Lewis $\mathrm{O}$, Wyatt MJ, et al: The distribution, clearance, and brainstem toxicity of panobinostat administered by convection-enhanced delivery. J Neurosurg Pediatr 22:288-296, 2018

66. Smith MA, Seibel NL, Altekruse SF, Ries LA, Melbert DL, O'Leary M, et al: Outcomes for children and adolescents with cancer: challenges for the twenty-first century. J Clin Oncol 28:2625-2634, 2010

67. Souweidane MM: Pontine tumors. J Neurosurg Pediatr 1:424-425, 2008

68. Souweidane MM, Kramer K, Pandit-Taskar N, Zhou Z, Haque S, Zanzonico P, et al: Convection-enhanced delivery for diffuse intrinsic pontine glioma: a single-centre, doseescalation, phase 1 trial. Lancet Oncol 19:1040-1050, 2018

69. Souweidane MM, Occhiogrosso G, Mark EB, Edgar MA: Interstitial infusion of IL13-PE38QQR in the rat brain stem. J Neurooncol 67:287-293, 2004
70. Souweidane MM, Occhiogrosso G, Mark EB, Edgar MA, Dunkel IJ: Interstitial infusion of carmustine in the rat brain stem with systemic administration of O6-benzylguanine. J Neurooncol 67:319-326, 2004

71. Vanan MI, Eisenstat DD: DIPG in children-what can we learn from the past? Front Oncol 5:237, 2015

72. Vykhodtseva N, McDannold N, Hynynen K: Progress and problems in the application of focused ultrasound for bloodbrain barrier disruption. Ultrasonics 48:279-296, 2008

73. Wagner S, Benesch M, Berthold F, Gnekow AK, Rutkowski $\mathrm{S}$, Sträter R, et al: Secondary dissemination in children with high-grade malignant gliomas and diffuse intrinsic pontine gliomas. Br J Cancer 95:991-997, 2006

74. Warren KE: Diffuse intrinsic pontine glioma: poised for progress. Front Oncol 2:205, 2012

75. Wu G, Broniscer A, McEachron TA, Lu C, Paugh BS, Becksfort $\mathrm{J}$, et al: Somatic histone $\mathrm{H} 3$ alterations in pediatric diffuse intrinsic pontine gliomas and non-brainstem glioblastomas. Nat Genet 44:251-253, 2012

76. Yoshimura J, Siu IM, Thomale UW, Jallo GI: The effects of temozolomide delivered by prolonged intracerebral microinfusion against the rat brainstem GBM allograft model. Childs Nerv Syst 28:707-713, 2012

77. Zhou Z, Ho SL, Singh R, Pisapia DJ, Souweidane MM: Toxicity evaluation of convection-enhanced delivery of smallmolecule kinase inhibitors in naïve mouse brainstem. Childs Nerv Syst 31:557-562, 2015

\section{Disclosures}

The authors report no conflict of interest concerning the materials or methods used in this study or the findings specified in this paper.

\section{Author Contributions}

Conception and design: both authors. Drafting the article: both authors. Critically revising the article: both authors. Reviewed submitted version of manuscript: both authors. Approved the final version of the manuscript on behalf of both authors: Souweidane.

\section{Correspondence}

Mark M. Souweidane: Weill Cornell Medical College, NewYorkPresbyterian Hospital, New York, NY. mmsouwei@med.cornell. edu. 